\title{
A Rare Use of a Shark's Fin? Pectoral Fin Scooping of a Sharksucker from The Flank Area of a Female Blacktip Shark
}

\author{
Erich K. Ritter* \\ Shark Research Institute / Florida Office, 5970 Osprey Place Pensacola, FL 32504, USA
}

\begin{abstract}
Sharks react in a variety of ways when sharksuckers attach to their bodies. Here, an unobserved behavior is described in which a blacktip shark, Carcharhinus limbatus, uses a pectoral fin in a scoop-like manner to specifically target the removal of a sharksucker, Echeneis naucrates, in the flank area. This coordinated use of the pectoral fin indicates an established bout in the shark's repertoire to remove sharksuckers. The anatomical flexibility and coordination of the fin is discussed; likewise, the potential origin and commonality within other species of the same family based on the rise of sharksuckers in the Eocene-Oligocene period. Special emphasis is placed on the value of a single observation of animal behavior in the field.
\end{abstract}

Keywords: Blacktip shark, Carcharhinus limbatus, irritation, pectoral fin, sharksucker.

\section{INTRODUCTION}

The pectoral fins of sharks are primarily used for body trim while swimming and steering [e.g., 1, 2] or occasionally among benthic shark species for moving over substrates [e.g., 3-5]. Less common is the use of pectoral fins in response to sharksucker irritation, e.g., while chafing, the rubbing of a shark's body on the ocean floor, a pectoral fin is used to enhance the rolling effect of the behavior by dragging it on the sea bottom [6].

This note describes the one-time observation of a second use of pectoral fins in response to sharksuckers, Echeneis naucrates, namely the brushing-off of a sharksucker from the flank of a female blacktip shark, Carcharhinus limbatus. The presented behavior, described here as scooping, was videotaped during a study of sharksucker irritation in Walker's Cay, Bahamas [6]. Scooping was recorded in Hi8Format (NTSC) and processed through Final Cut ${ }^{\circledR}$ Pro 6.x by Apple ${ }^{\circledR}$. Single frames were transferred to Adobe ${ }^{\circledR}$ Photoshop CS3 for further analysis. Tailbeat frequency, measured as a fraction of tailbeat cycle per second (tbs), was used as a relative measurement for speed [e.g., 7-9]. The average of three consecutive tailbeat cycles was measured five times over the entire 27 -second observation period while the shark was videotaped. The relative swim speed remained at $0.5 \pm 0.05$ tbs up to and including scooping, with a brief increase to approximately $1.0 \pm 0.05$ tbs immediately after scooping. Since the speed increase only lasted one tailbeat cycle, an approximation of the two half cycles was used. For relative speed comparison, the tailbeat frequencies of five randomly chosen blacktip sharks of similar size from the same dive, which did not carry sharksuckers, were also taken. Their relative swim speed averaged $0.6 \pm 0.1$ tbs.

*Address correspondence to this author at the Shark Research Institute / Florida Office, 5970 Osprey Place Pensacola, FL 32504, USA;

Tel: 786301 3310; Fax: +850 473 1294; E-mail: erichritter@att.net
The sharksucker remained attached for approximately 12 seconds on the shark's lower right flank between the pectoral and pelvic fin without triggering any visible reaction from the shark. The sharksucker then moved on to the very position that later triggered scooping. The change of the sharksucker's position created an immediate, albeit minor, tilt in rotation of approximately $10^{\circ}$ along the shark's horizontal axis that last for about four seconds without a further reaction from the teleost. While in a tilted position, the shark maintained a swim speed of $0.5 \pm 0.05$ tbs. For another eight seconds, the shark continued a regular subundulatory swimming motion before scooping commenced (Fig. 1a). The scooping behavior started with an increase in lateral bending along the vertical axis of the shark, combined with a simultaneous posterolateral motion of the right pectoral fin (Fig. 1b). At maximum body curvature (Fig. 1c), the pectoral fin is abducted, leading to an immediate change in position of the sharksucker (Fig. 1d). During the adducting of the fin (Fig. 1e), the sharksucker briefly lost body contact, but reattached itself immediately and then moved towards the shark's head area. Scooping ended with the pectoral fin's forward motion and reverse of lateral bending (Fig. 1f).

The right pectoral fin showed slight irregular flickering between tilting and scooping. Flickering refers to a diffuse twitching of the flank muscles, often accompanied by shaking of one pectoral fin, but less so among other fins. This motion is comparable to the effect in mammals of panniculus camosus muscle twitching when irritated by insects or when shivering [e.g., 10-12].

In general, a shark's response to sharksucker irritation is described in one of two ways. One, a shark uses some type of surface to chafe off the sharksucker, e.g., the seafloor [6], or the water surface through leaping out of the water and falling back onto the irritated area [13]; or, two, the shark contorts its body to manipulate the affected skin area, or uses diffuse skin-twitching to motivate a sharksucker to attach itself elsewhere [14]. When manipulating affected areas by 

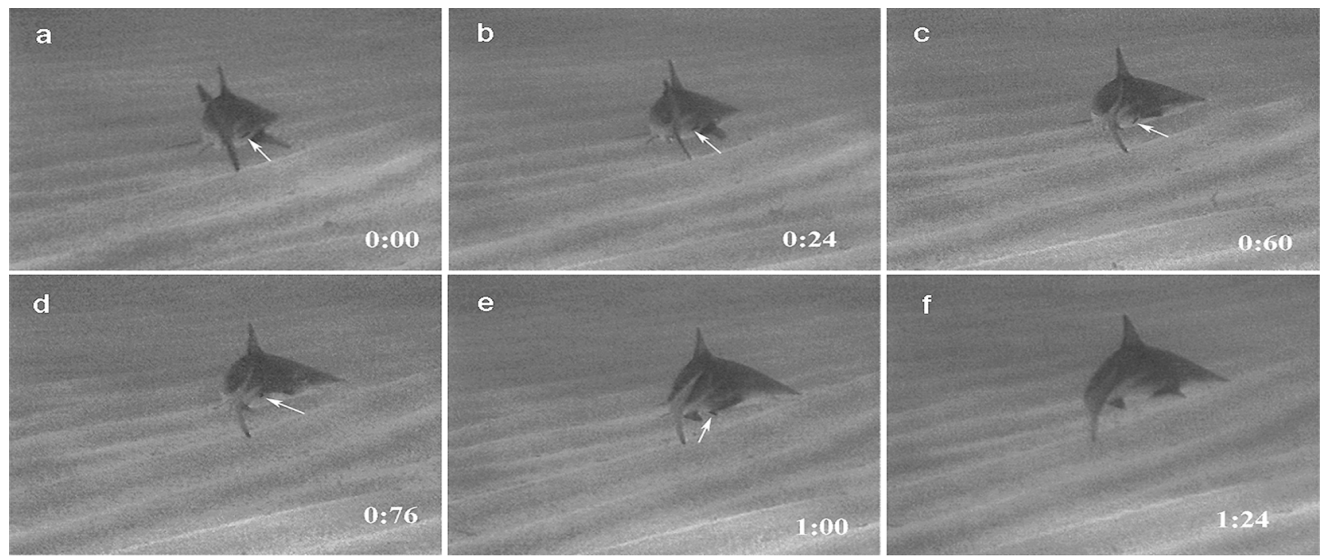

Fig. (1). Scooping off a sharksucker from the flank area. Arrow shows sharksucker position. Time reflects approximation based on 25 frames $\sec ^{-1}$.

squeezing together or stretching the skin to reduce suction pressure, a shark often simultaneously lifts its snout and lowers its pectoral fins in a process called winding [14]. This is frequently misinterpreted as an agonistic display $[15,16]$. Scooping is part of the second category of body-only patterns; however, compared to other motions within this group, it is very limited and entirely depends on the reach of the pectoral fin and body flexibility. This very restricted use could likely reflect the scarce observation.

During this observation, the sharksucker caused the shark to react twice. It is unclear why a motion interruption of approximately eight seconds occurred between the initial tilting reaction and scooping. One explanation could be that the initial irritation was minor, but the suction pressure then gradually increased [e.g., 17] and lead to a second reaction. This could occur if the sharksucker used a higher suction pressure due to an increase in shark speed. Alternatively, relocation of the sharksucker to a different position on the shark demanded increased suction due to a change of the denticle surface [18]. Although increases in swim speed as a reaction to sharksucker irritation have previously been observed [19], speed comparison with the other cruising blacktip sharks showed no difference. A second explanation for the reaction pause could be that the shark was just perching in anticipation of an additional move by the sharksucker, either to a less irritating spot or towards the shark's head area, which functions as a tactile signal for cleaning purposes (unpubl. data).

To successfully scoop, pectoral fins need to be flexible. These fins are plesodic with a strong support from the cartilaginous radials and ceratotrichia to stiffen the fin [20]. In contrast, the rim along the inner and posterior margin, and its immediate fringe area, remain flexible - a prerequisite for scooping to follow the curvature of the flank area. The range of pectoral fin along the flank depends not only on flexibility and controlled use of the dorsal and ventral pterygoidei muscles, but also on body suppleness along the vertical axis. Additionally, the scoop motion required counterbalance, indicated by the slight change in the main axis of the caudal fin [e.g., 21, 22].

Despite the motion complexity and novelty, the significance of a one-time observation of a behavior needs to be valued. One of the prime aspects of observing animals in the field is accessibility [e.g., 23], as some species are nearly impossible to observe. Additionally, it is well known that studying sharks in the field entails many limitations, clearly reflected by the rather poor knowledge of their overall behavior [e.g., 24-26]. From this viewpoint, even a single observation carries importance by revealing new insights into a behavioral aspect. However, the full value of such an observation will likely not be seen until more individual blacktip sharks, or other species, are observed performing this behavior. It is quite certain that other Carcharhinid species likely possess scooping in their behavioral repertoire. Assuming enough time is given to observe those species, this behavior will be noticed in due time.

Using fossil records, scooping might have been present for a long period of time. Early sharksucker fishes of the family Echeneidae first appeared in the Eocene-Oligocene period [e.g., 27, 28], a time where Carcharhinid sharks were already widespread [e.g., 29]. That makes it very plausible that recent descendants are indeed adjusted to behavioral patterns in response to each other. To what extent other shark species react to the same irritation along their flanks is unknown, but considering the well-developed event of scooping, and the long co-existence between Echeneid fishes and Carcharhinid sharks, similar bouts can be expected.

\section{CONFLICT OF INTEREST}

None declared.

\section{ACKNOWLEDGEMENT}

None declared.

\section{REFERENCES}

[1] Fish FE, Shannahan LD. The role of the pectoral fins in body trim of sharks. J Fish Biol 2000; 56: 1062-73.

[2] Wilga CD, Lauder GV. Three-dimensional kinematics and the wake structure of the pectoral fin during locomotion in leopard sharks Triakis semifasciata. J Exp Biol 2000; 203: 2261-78.

[3] Pridmore PA. Submerged walking in the epaulette shark Hemiscyllium ocellatum (Hemiscyllidae) and its implications for locomotion in rhipidistian fishes and early tetrapods. Zoology 1995; 98: 27897.

[4] Goto T, Nishida K, Nakaya K. Internal morphology and function of paired fins in the epaulette shark, Hemiscyllium ocellatum. Ichthyol Res 1999; 46: 281-7. 
[5] Wilga CD, Lauder GV. Functional morphology of the pectoral fins in bamboo sharks, Chiloscyllium plagiosum: Benthic vs. Pelagic station-holding. J Morphol 2001; 249: 195-209.

[6] Ritter E. Use of sand ripples to enhance chafing in Caribbean reef sharks (Carcharhinus perezi) and blacktip sharks (Carcharhinus limbatus). Bull Mar Sci 2011; 87: 413-9.

[7] Dewar H, Graham J. Studies of tropical tuna swimming performance in a large water tunnel - energetics. J Exp Biol 1994; 192: 1331.

[8] Lowe CG. Kinematics and critical swimming speed of juvenile scalloped hammerhead sharks. J Exp Biol 1996; 199: 2605-10.

[9] Ritter E. Measuring swim speeds of sharks in the vicinity of humans. Baham Nat J Sci 2007; 2: 23-6.

[10] Miller AD. Localization of motoneurons innervating individual abdominal muscles of the cat. J Comp Neurol 1987; 256: 600-6.

[11] Mayumi H, Nomoto K, Good RAA. A surgical technique for experimental free skin grafting in mice. Surg Today 1988; 18: 54857.

[12] Blight AR. Morphometric analysis of a model of spinal cord injury in guinea-pigs with behavioral evidence of delayed secondary pathology. J Neurol Sci 1991; 103: 156-71.

[13] Ritter EK, Brunnschweiler JM. Do sharksuckers, Echeneis naucrates, induce jump behaviour in blacktip sharks, Carcharhinus limbatus? Mar Freshw Behav Physiol 2003; 36: 111-3.

[14] Ritter E. Analysis of sharksucker, Echeneis naucrates, induced behavior patterns in the blacktip shark, Carcharhinus limbatus. Environ Biol Fish 2002; 64: 111-5.

[15] Johnson RH, Nelson DR. Agonistic display in the gray reef shark, Carcharhinus menissorah, and its relationship to attacks on man. Copeia 1973; 1973: 76-84.

[16] Ritter EK, Godknecht AJ. Agonistic displays in the blacktip shark Carcharhinus limbatus. Copeia 2000; 2000: 282-4.

[17] Fulcher BA, Motta PJ. Suction disk performance of echeneid fishes. Can J Zool 2006; 84: 42-50.
[18] Raschi W, Tabit C. Functional aspects of placoid scales: a review and update. Mar Freshw Res 1992; 43: 123-47.

[19] Brunnschweiler J. Sharksucker-shark interaction in two carcharhinid species. Mar Ecol 2006; 27: 89-94.

[20] Compagno LJV. Sharks of the order of Carcharhiniformes. Princeton: Princeton University Press 1988.

[21] Lauder GV. Function of the caudal fin during locomotion in fishes: Kinematics, flow visualization, and evolutionary patterns. Am Zool 2000; 40: 101-22.

[22] Wilga CD, Lauder GV. Function of the heterocercal tail in sharks: quantitative wake dynamics during steady horizontal swimming and vertical maneuvering. J Exp Biol 2002; 205: 2365-74.

[23] Lehner PN. Handbook of ethological methods. $2^{\text {nd }}$ ed. Cambridge: Cambridge University Press 1996.

[24] Klimley AP. The secret life of sharks. New York: Simon \& Schuster 2003.

[25] Castro JI. The sharks of North America. Oxford: Oxford University Press 2011.

[26] Sims DW. Tracking and analysis techniques for understanding free ranging shark movement and behavior. In: Carrier JC, Musick JA, Heithaus MR, Eds. Sharks and their relatives II. Biodiversity, adaptive physiology and conservation. Boca Raton, FL: CRC Press 2010; pp. 351-92.

[27] Micklich N. New information on the fish fauna of the Frauenweiler fossil site. Ital J Zool 1998; 65: 169-84.

[28] O'Toole B. Phylogeny of the species of the superfamily Echeneoidea (Perciformes: Carangoidei: Echeneidae, Rachycentridae, and Coryphaenidae), with an interpretation of echeneid hitchhiking behaviour. Can J Zool 2002; 80: 596-623.

[29] Kriwet J. Additions to the eocene selachian fauna of Antarctica with comments on the Antarctic selachian diversity. J Vertebr Paleontol 2005; 25: 1-7.

(c) Erich K. Ritter; Licensee Bentham Open.

This is an open access article licensed under the terms of the Creative Commons Attribution Non-Commercial License (http://creativecommons.org/licenses/ by-nc/3.0/) which permits unrestricted, non-commercial use, distribution and reproduction in any medium, provided the work is properly cited. 